\title{
Element Abundances in Magellanic Cloud H II Regions from Carbon to Argon ${ }^{1}$
}

\author{
Donald R. Garnett \\ Astronomy Dept., University of Minnesota, Minneapolis, MN 55455 and \\ Steward Observatory, University of Arizona, Tucson, AZ 8521
}

\begin{abstract}
I review measurements of heavy element abundances within $\mathrm{H}$ II regions in the Magellanic Clouds, highlighting in particular improved determinations of carbon abundances based on UV spectroscopy with Hubble Space Telescope. In general, the Magellanic Cloud H II regions show average underabundances in $\mathrm{O}, \mathrm{Ne}$, and $\mathrm{S}$ (relative to their Galactic counterparts) that are similar to those measured in Magellanic Cloud stars. However, comparison of stars and ionized gas shows discrepancies in $\mathrm{C}$ and $\mathrm{N}$ abundances that may be related to recently recognized mixing processes that may be operating in massive stars.
\end{abstract}

\section{Introduction}

Spectroscopy of photoionized nebulae provides a very useful probe of the gas phase composition of the interstellar medium (ISM), an important observational constraint for models of galaxy evolution. One motivation for studying the ionized gas phase is trivial: many $\mathrm{H}$ II regions are very luminous, and so the emission-line spectroscopy is relatively easy to obtain. This means that H II regions typically provide our first measurements of element abundances in galaxies other than our own, and this was the case for the Magellanic Clouds. More seriously, spectroscopy of $\mathrm{H}$ II regions probes the interstellar gas on a wide range of scales, from kiloparsecs down to sub-parsec scales (in the Magellanic Clouds). This allows one to analyze the homogeneity of the ISM on a variety of scales and thus to address questions of mixing and transport of stellar-enriched material in the ISM. The composition of the ISM is also a fundamental parameter in the determination of important ISM physical quantities such as the cooling function of the gas, the conversion factor from $\mathrm{CO}$ intensity to molecular hydrogen column density, the dust-to-gas ratio of the ISM, etc. For such issues the composition of the gas itself is more useful than the stellar abundances. Third, a comparison of the ISM composition with that of young stars can provide illumination on physical processes which may be operating in stars and gas, for example, mixing processes within stars (see Venn, this volume), or the composition of interstellar

\footnotetext{
${ }^{1}$ Based in part on observations with the NASA/ESA Hubble Space Telescope obtained at the Space Telescope Science Institute, which is operated by the Association of Universities for Research in Astronomy, Inc., under NASA contract NAS5-26555.
} 
dust grains. Studies of abundances in both the ISM and in stars are thus highly complementary.

\section{Abundance Data}

\subsection{Published Surveys}

Table 1 lists the sources of $\mathrm{H}$ II region spectroscopy used to prepare this review. A few things can be noted about this list. First, a great deal of spectroscopic data has been accumulated for some $20 \mathrm{H}$ II regions each in the LMC and SMC. At the same time, one can see that, with few exceptions, the sources are 15-25 years old, and little recent work has been done using efficient long-slit CCD spectrographs. This is a bit of a shame, since improved instrumentation often leads to new discoveries in familiar objects. In particular, the Magellanic Clouds, because of their well-constrained distances and low interstellar reddening, provide fertile grounds for comparing the observed spectrum with predictions of photoionization models and computed stellar atmospheres (e.g., see Oey \& Massey 1995, Wilcots 1994a,b). Little mid-IR and far-IR spectroscopy has been done for H II regions in the Clouds as well, and we eagerly await the results of the ISO programs doing spectroscopy of $\mathrm{H}$ II regions in the clouds, examples of which have been shown at this conference (Thornley et al., Monetti et al., this volume).

Table 1. 'Bibliography of LMC and SMC H II Region Spectroscopy

\begin{tabular}{ll}
\hline Reference & Notes \\
\hline Peimbert \& Torres-Peimbert 1974, 1976 & \\
Dufour 1975 & \\
Dufour \& Harlow 1977 & \\
$\begin{array}{l}\text { Pagel, Edmunds, Fosbury, \& Webster 1978 } \\
\text { Dufour, Shields, \& Talbot 1982 }\end{array}$ & \\
$\begin{array}{l}\text { Dennefeld \& Stasińska 1983 } \\
\text { Heydari-Malayeri et al. 1985-1988 }\end{array}$ & \\
Mathis, Chu, \& Peterson 1985 & \\
Rosa \& Mathis 1987 & 30 Doradus \\
Russell \& Dopita 1990 & 30 Doradus \\
\hline \hline
\end{tabular}

Average LMC and SMC interstellar abundances for elements determined from optical spectroscopy $(\mathrm{O}, \mathrm{N}, \mathrm{Ne}, \mathrm{S}, \mathrm{Ar})$ are essentially unchanged from the last review by Dufour (1990). For the rest of this review, therefore, I will concentrate on a discussion of more recent work.

\subsection{A Detailed Spatially-Resolved Study of 30 Doradus}

30 Doradus is certainly the best-studied H II region in the Magellanic Clouds, because it is the nearest example of a giant $\mathrm{H}$ II region and because of the many interesting stellar and interstellar phenomena found within the region. Some early detailed spectroscopic studies of 30 Dor hinted that the region was quite homogeneous in its composition (Mathis, Chu \& Peterson 1985; Rosa \& Mathis 
1987). Perhaps the ultimate study can be found in the thesis of Kinkel (1993), who analyzed new and previously published spectra for 76 separate spatial positions in 30 Dor, including positions outside the bright filaments. Kinkel's work on 30 Doradus can be considered a benchmark study in the field of giant H II regions and his analysis of the 30 Dor spectra uncovered a number of noteworthy features:

1. There is an electron temperature gradient within 30 Doradus, in the sense that the derived [O III] temperature systematically increases as the fraction of oxygen in the form $\mathrm{O}^{+2}$ decreases. Such a variation can be understood as a combination of "radiation hardening" and less efficient cooling in the $\mathrm{O}^{+}$zone, leading to higher $T_{e}$ in the singly-ionized zone. The result is that the derived oxygen abundance is correlated with $\mathrm{O}^{+}$fraction if a single average value for $T_{e}$ is assumed. Taking into account the temperature gradient leads to uniform derived $\mathrm{O} / \mathrm{H}$ across the nebula. However, the difference in $\mathrm{O} / \mathrm{H}$ between the two cases is small, only 0.03 dex for 30 Doradus.

2. Derived abundances for $\mathrm{S}$ and Ar using standard ionization corrections for $\mathrm{Ar}^{+3}$ and $\mathrm{S}^{+3}$, correlate with $\mathrm{O}^{+} / \mathrm{O}$, in the sense that the standard corrections appear to predict too much $\mathrm{Ar}^{+3}$ and $\mathrm{S}^{+3}$ in high-ionization regions. This may indicate problems with ionization cross sections or stellar atmosphere fluxes at high photon energies, and this remains a problem for future study.

3. An apparent enhancement of heavy element abundances at one position in 30 Doradus, reported by Mathis \& Rosa (1987), was not confirmed.

The results of Kinkel's analysis show that the derived abundances across 30 Dor are remarkably uniform: $\log \mathrm{O} / \mathrm{H}=-3.71$, with an $\mathrm{rms}$ dispersion of only \pm 0.043 dex. Based on this uniformity, Kinkel places an upper limit of $t^{2}<10^{-3}$ (in terms of Peimbert's $t^{2}$ parameter) for electron temperature fluctuations. These results place severe limits on the effects of shocks on the observed spectra of giant H II regions, and on the pollution of the H II region by supernova ejecta.

\subsection{Carbon in $\mathbf{H}$ II Regions from HST Observations}

Measurements of carbon in $\mathrm{H}$ II regions have been much more limited than for oxygen, because the common ionization states have no strong optical emission lines; the strongest $\mathrm{C}^{+}$transitions are in the UV or far-IR, while the strongest transitions of $\mathrm{C}^{+2}$ are in the UV. The IUE satellite permitted the first survey of carbon abundances in Magellanic Cloud H II regions (Dufour, Shields, \& Talbot 1982), indicating significantly reduced $\mathrm{C} / \mathrm{O}$ abundances ratios compared to the Galaxy. On the other hand, measurements of $\mathrm{C}$ in cool supergiants in the Clouds indicated much higher carbon abundances than the IUE results (Spite, Barbuy, \& Spite 1989).

UV spectroscopy with Hubble Space Telescope greatly improves our ability to measure accurate $\mathrm{C} / \mathrm{O}$ ratios in $\mathrm{H}$ II regions. The region $1600-2000 \AA$ contains a very nice series of emission lines from $\mathrm{C}^{+2}, \mathrm{O}^{+2}, \mathrm{~N}^{+2}, \mathrm{Ne}^{+2}$, and $\mathrm{Si}^{+2}$, with similar excitation energies; similarly, the region $2100-2500 \AA$ includes transitions 
from $\mathrm{C}^{+}, \mathrm{O}^{+}$, and $\mathrm{N}^{+}$. Measurements of these $\mathrm{UV}$ lines together allow one to derive abundance ratios for these elements with small uncertainties from differential reddening and temperature fluctuations. My collaborators and I have been obtaining UV spectra in extragalactic H II regions, including two in the LMC and one in the SMC, to derive carbon abundances with greatly reduced uncertainties.

Our C abundances for the LMC and SMC based on HST data are listed in Table 2. The LMC value is the average for 30 Dor (Garnett et al. 1995) and N44C (Garnett, Galarza, \& Chu 1999), while the SMC value is a single measurement for N88A (Kurt et al. 1999). Note that the SMC-N88A value is about 0.3 dex higher than the average value for SMC regions derived from IUE measurements (see Dufour 1990); it is not clear if this is a real difference (perhaps due to variations in grain abundances), or if it is due to systematic uncertainties. More $H S T$ UV spectroscopy is needed to understand this difference. Nevertheless, our measurements so far indicate with high confidence that $\mathrm{C}$ is significantly underabundant with respect to $\mathrm{O}$ in the SMC, compared to the Galaxy, while in the LMC C/O is at most slightly lower than in the solar neighborhood, taking into account a possible modest correction for $\mathrm{C}$ and $\mathrm{O}$ in dust grains.

\section{Average H II Region Abundances and Comparison with Stars}

Average heavy element abundances for $\mathrm{H}$ II regions in the Magellanic Clouds from optical/UV spectroscopy are listed in Table 2.

Table 2. Average H II Region Abundances in the Magellanic Clouds

\begin{tabular}{cccccccc}
\hline Galaxy & $\log \mathrm{O} / \mathrm{H}$ & $\log \mathrm{C} / \mathrm{H}$ & $\log \mathrm{N} / \mathrm{H}$ & $\log \mathrm{Ne} / \mathrm{H}$ & $\log \mathrm{S} / \mathrm{H}$ & $\log \mathrm{Ar} / \mathrm{H}$ & $\log \mathrm{Si} / \mathrm{H}$ \\
\hline LMC & -3.6 & -4.1 & -5.1 & -4.4 & -5.3 & -5.8 & -5.3 \\
SMC & -4.0 & -4.6 & -5.5 & -4.8 & -5.7 & -6.1 & -5.7 \\
\hline \hline
\end{tabular}

The element underabundances in the Clouds relative to the Galaxy are shown in Figure 1 as the filled circles, along with a comparison to values for various kinds of stars in the Magellanic Clouds. For this figure I compare Magellanic Cloud objects to their solar neighborhood counterparts, i.e., LMC H II regions vs. the Orion Nebula (Esteban et al. 1998), SMC B stars vs. solar neighborhood B stars, etc. This follows the practice of Pagel (1993).

Figure 1 shows that the differential abundances for $\mathrm{O}, \mathrm{Ne}$, and $\mathrm{S}$ are in good agreement for both stars and $\mathrm{H}$ II regions (no stellar data is available for $\mathrm{Ar}$, which shows the same underabundance as $\mathrm{O}-\mathrm{Ne}-\mathrm{S}$ in the $\mathrm{H}$ II regions). Silicon shows a curious enhancement in the $\mathrm{H}$ II regions relative to stars; however, $\mathrm{Si}$ suffers density-dependent depletion onto grains, and this differential comparison with Orion may not reflect the true abundance difference.

On the other hand, the $\mathrm{C}$ and $\mathrm{N}$ abundances show significant differences between the $\mathrm{H}$ II regions and the young stars. $\mathrm{C}$ in the $\mathrm{H}$ II regions seems to be in good agreement with the B stars and A supergiants in both the LMC and SMC, but disagrees significantly with the cool supergiants in the SMC and disagrees marginally with the LMC cool supergiants. Unlike $\mathrm{Si}, \mathrm{C}$ shows no 

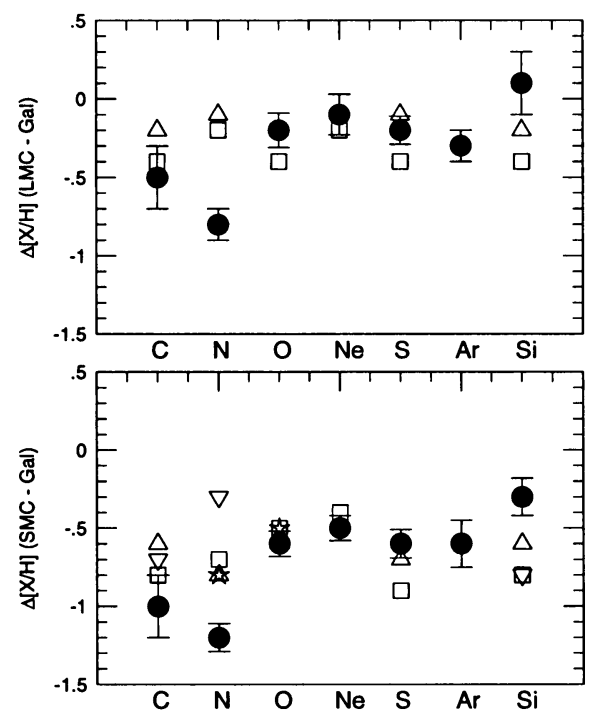

Figure 1. Comparison of $\mathrm{H}$ II region abundances with stellar abundances in the Magellanic Clouds. Filled circles: $\mathrm{H}$ II regions. Open squares: main sequence B stars (Rolleston et al. 1996; Lennon et al. 1996). Open triangles: cool supergiants (Hill et al. 1997; Luck et al. 1998). Stars: A supergiants (Venn 1999).

evidence for environment-dependent depletion (Sofia et al. 1997), so we assume that the differential abundance for the H II regions is correct. Nitrogen shows even greater discrepancies between the stars and gas, in the sense that the stars are systematically overabundant in $\mathrm{N}$ by up to a factor of 10 compared to the $\mathrm{H}$ II regions. Since nitrogen is not readily incorporated into grains (Mathis 1996), depletion effects can not be invoked to explain this discrepancy. The discrepancy with the H II regions increases as one goes from B stars to A supergiants to cool supergiants.

The differences in $\mathrm{C}$ and $\mathrm{N}$ may reflect mixing processes occurring inside massive stars, such as rotational mixing as discussed by Fliegner, Langer, \& Venn (1996) and by Venn at this conference. At the same time, with a limited amount of UV data for the H II regions it is probably premature to draw firm conclusions. UV spectra for more H II regions in the Clouds are needed to rule out significant spatial variations in the carbon abundance. Deeper exposures are needed to detect the N III] $1750 \AA$ emission multiplet, which will yield definitive nitrogen abundances. Future observations of both $\mathrm{H}$ II regions and stars with $H S T$ and large ground-based telescopes will yield tremendous insight into the properties of both stars and gas in the Clouds.

Acknowledgments. The work on carbon abundances in $\mathrm{H}$ II regions is supported in part by NASA-LTSARP grant NAG5-6416. Thanks go to Michael Rosa for providing me a copy of Ulrich Kinkel's fine PhD thesis. I also thank Kim 
Venn for vivid discussions of stellar abundances in the Magellanic Clouds, and for pointing out a misunderstanding in my evaluation of the stellar abundances.

\section{References}

Dennefeld, M., \& Stasińska, G. 1983, A\&A, 118, 234

Dufour, R.J. 1975, ApJ, 195, 315

Dufour, R.J. 1990, in Evolution in Astrophysics, ESA SP-310, 117

Dufour, R.J., \& Harlow, W.V. 1977, ApJ, 216, 706

Dufour, R.J., Shields, G.A., \& Talbot, R.J. 1982, ApJ, 252, 461

Esteban, C., et al. 1998, MNRAS, 295, 401

Fliegner, J., Langer, N., \& Venn, K.A. 1996, A\&A, 308, L13

Garnett, D.R., et al. 1995, ApJ, 443, 64

Garnett, D.R., Galarza, V., \& Chu, Y.-H. 1999, in preparation

Heydari-Malayeri, M., \& Testor, G. 1985, A\&A, 144, 98

Heydari-Malayeri, M., \& Testor, G. 1986, A\&A, 162, 180

Heydari-Malayeri, M., Niemela, V.S., \& Testor, G. 1987, A\&A, 184, 300

Heydari-Malayeri, M., Le Bertre, T., \& Magain, P. 1988, A\&A, 195, 230

Hill, V., Barbuy, B., \& Spite, F. 1997, A\&A, 323, 461

Kinkel, U. 1993, $\mathrm{PhD}$ thesis, University of Heidelberg

Kurt, C.M., et al. 1999, ApJ, 518, 246

Lennon, D.J., et al. 1996, A\&A, 314, 243

Luck, R.E., Moffett, T.J., Barnes, T.G. III, \& Gieren, W.P. 1998, AJ, 115, 605 Mathis, J.S. 1996, ApJ, 472, 643

Mathis, J.S., Chu, Y.-H., \& Peterson, D.E. 1985, ApJ, 292, 155

Oey, M.S., \& Massey, P. 1995, ApJ, 452, 210

Pagel, B.E.J. 1993, in New Aspects of Magellanic Cloud Research, B. Baschek, G. Klare, and J. Lequeux, Berlin: Springer-Verlag, 330

Pagel, B.E.J., et al. 1978, MNRAS, 184, 569

Peimbert, M., \& Torres-Peimbert, S. 1974, ApJ, 193, 327

Peimbert, M., \& Torres-Peimbert, S. 1976, ApJ, 203, 581

Rolleston, W., et al. 1996, A\&A, 315, 95

Rosa, M.R., \& Mathis, J.S. 1987, ApJ, 317, 163

Russell, S.C., \& Dopita, M.A. 1990, ApJS, 74, 93

Sofia, U.J., Cardelli, J.A., Guerin, K.P., \& Meyer, D.M. 1997, ApJ, 482, 105

Spite, M., Barbuy, B., \& Spite, F. 1989, A\&A, 222, 35

Venn, K.A. 1999, ApJ, 518, 405

Wilcots, E. 1994a, AJ, 107, 1338

Wilcots, E. 1994b, AJ, 108, 1674 


\section{Discussion}

Giuseppe Bono: Did you estimate the helium-to-metal enrichment ratio?

Garnett: I did not look at He. The helium abundances will require new corrections for collision excitation; current estimates for electron densities are not adequate to do this accurately. Because we know that $\mathrm{C}$ and $\mathrm{N}$ abundances are under-abundant with respect to $\mathrm{O}$, the $\Delta \mathrm{Y} / \Delta \mathrm{Z}$ relation will be slightly steeper, but not very much. 